\title{
Ex Vivo Implantation Study of Minimally Invasive Glaucoma Drainage Device
}

\author{
Jeffrey Chun-Hui Lin ${ }^{1, *}$, Feiqiao $\mathrm{Yu}^{1}$, Saloomeh Saati ${ }^{2}$, Rohit Varma ${ }^{2}$, Mark S. Humayun ${ }^{2}$, Yu-Chong Tai ${ }^{1}$ \\ ${ }^{1}$ Department of Electrical Engineering, California Institute of Technology, Pasadena, CA, USA \\ ${ }^{2}$ Doheny Eye Institute, Keck School of Medicine of the University of Southern California, Los Angeles, CA, USA. \\ *Contact author's email: linch@mems.caltech.edu
}

\begin{abstract}
We present in this paper the first ex vivo implantation results of our minimally invasive glaucoma drainage device (GDD.) The GDD is designed to treat glaucoma patients by draining out their extraneous aqueous humor out of the anterior chamber utilizing a MEMS micro-fluidic normally closed (NC) check valve. The NC check valve is encapsulated in protective tubing made from parylene $\mathrm{C}$, which has been proved to be biocompatible in implantation. A new packaging and a benchtop testing procedure is established to characterize the integrated GDD prior to its implantation into enucleated porcine eyes. Preimplanted characterization curve demonstrates a cracking pressure of $10-20 \mathrm{mmHg}$ of the $\mathrm{NC}$ check valve, which agrees with our theoretical design. Ex vivo implantation results show that cracking pressure is measured as $24 \mathrm{mmHg}$ by unloading the eye pressure. The little offset of the cracking pressure comes from the differences between the in vitro and $e x$ vivo testing environments. The hysteresis behavior of the $\mathrm{NC}$ check valve is also examined during implantation and is presented here.
\end{abstract}

Keywords - glaucoma; micro check valves; microfluidics; paryelene; microelectromechanical systems

\section{INTRODUCTION}

Glaucoma is a kind of eye disease where aqueous humor produced by the ciliary body cannot be drained out normally by patients' eyes themselves. Without successful eye fluid drainage, the liquid will accumulated in the anterior chamber of the eye, rising eye pressure. The elevated pressure will then be transmitted onto the retina in the back of the eye ball and damage the optic nerve, causing a tunnel view of and may lead to total blindness if not treated well. Current major medication for glaucoma includes eye drops which either reduces the eye fluid production rate or increase the drainage rate. However, eye drops may accompany several side effects such as blurred vision, low blood pressure, and fluctuation in heart rhythm [1]. In addition, it also comes with compliance issues since some patients could be refractory to all medications. Glaucoma drainage device has been developed as an alternative solution to treat glaucoma patients who are resistant to normal glaucoma medications. A successful GDD can drain out aqueous humor accumulated inside the anterior chamber continuously and lower the intraocular pressure (IOP) to within 10-20 mmHg. We previously presented a parylene-tube-type GDD paradigm incorporating either one NC check valve or dual NC with normally open (NO) check valves that regulate intraocular pressure (IOP) passively with no power consumption [2]-[3]. The biocompatible parylene tube of the GDD can be minimal invasively implanted under the conjunctiva using a gauge \#19 hypodermic needle, as shown in Figure 1. The key component of the GDD is one NC check valve which is designed to open at $10-20 \mathrm{mmHg}$, allowing extraneous aqueous humor to flow out of anterior chamber when the IOP is higher than the critical pressure. However, due to different environmental conditions in vivo and in vitro, successful bench-top results are insufficient to predict the GDD's Ex vivo and in vivo behaviors. Here we present a study of the Ex vivo implantation of the GDD, discussing the packaging and testing procedure before the surgery, and also the results after the implantation. An enucleated porcine eye is used as our implantation model in the process and a heightadjustable saline bottle is used to mimic eye fluid and simulate different eye pressure situations.

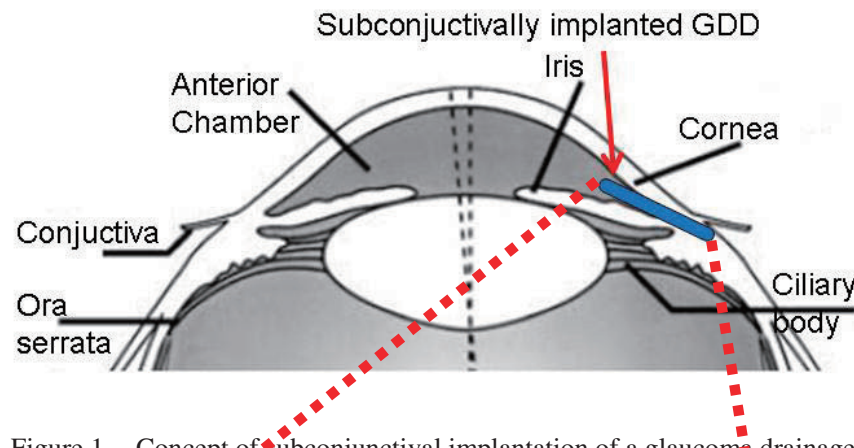

Figure 1. Concept of subconjunctival implantation of a glaucom drainage device. The overad implantation is done using hypodermic needle incision.

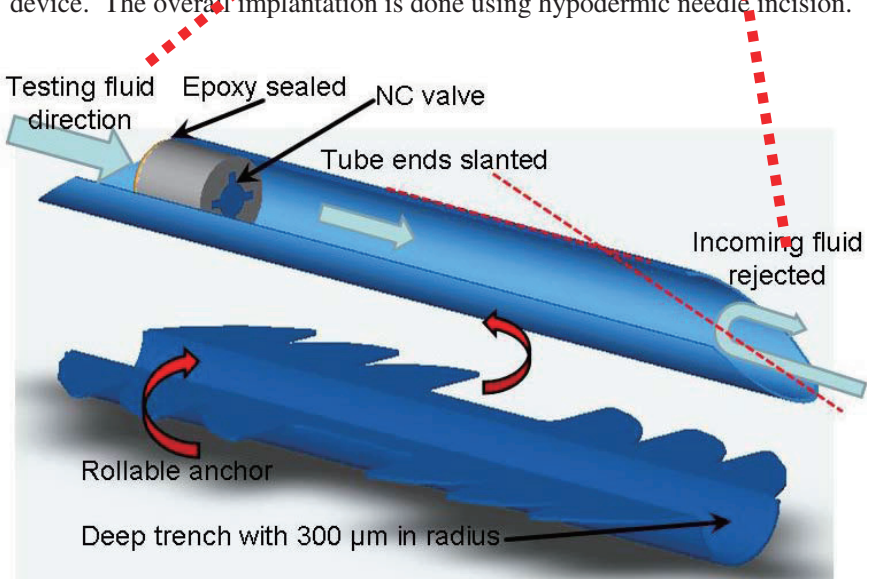

Figure 2. Glaucoma drainage device configuration. It comprises one Normally-Closed (NC) check valve, one parylene micro tube and that mounted onto a rollable parylene anchor. 


\section{DEVICE DESIGN}

A GDD configuration is shown in Figure 2. It comprises three major parts: one NC check valve, one parylene micro tube and one rollable parylene anchor.

\section{A. Design of $N C$ check valve}

The NC check valve remains closed as long as eye pressure is lower than the designed cracking pressure. It restricts eye fluid from leaking out of the anterior chamber, which otherwise causes hypotony. Owing to the inherent structure of the NC valve, fluid coming from the opposite way will be rejected, protecting the anterior chamber from any unwanted fluid flowing in. The cross section of the NC check valve is shown in Figure 3. The necessary pre-stress is introduced by stiction adhesion after the drying process, which occurs between parylene and silicon surface, and pulls down the tethers. The cracking pressure can be controlled by many parameters such as height of the stiction part of the parylene, parylene thickness, tether numbers, and the geometry of tethers. The flow rate is defined by the size of the opening orifice and the opening gap of the parylene membrane. The proper geometry of check valves was designed and simulated by finite element analysis software, COSMOS, as shown in Figure 4. Because eye pressure is measured as $10-20 \mathrm{mmHg}$ for normal people, 15 $\mathrm{mmHg}$ is picked up as the cracking pressure during its simulation. The outer diameter (OD) of the check valve is designed to be $500 \mu \mathrm{m}$ so that the overall GDD size can be fit into gauge \#19 needle, which inner diameter (ID) is $690 \mu \mathrm{m}$.

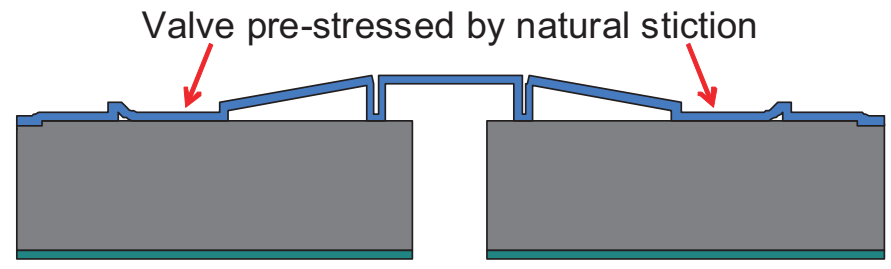

Figure 3. Cross-section of a Normally-Closed (NC) check valve. The valve is pre-stressed by natural stiction which inevitably happens after drying process.

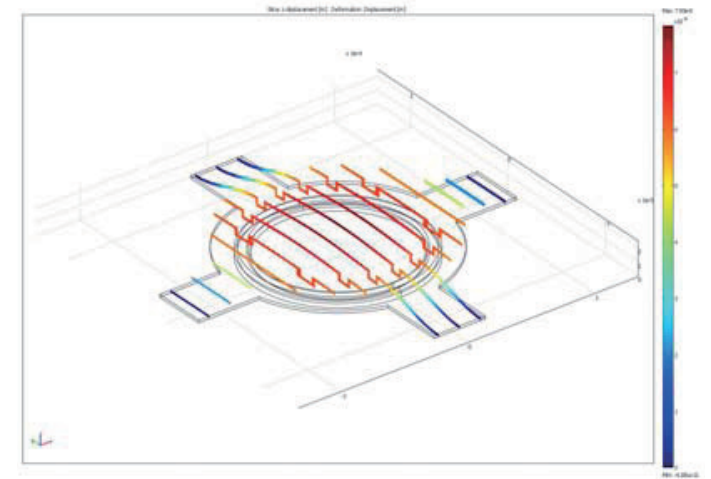

Figure 4. COSMOS mechanical analysis of normally-cloased check valves

\section{B. Design of parylene micro tube}

There are 2 main issues of the accommodation tube to concern about: the biocompatibility and the size. In terms of the biocompatibility, parylene $\mathrm{C}$ (poly para-xylylene $\mathrm{C}$ ) is used as our micro tube material. Parylene $\mathrm{C}$ is classified in FDA's USP class VI and is totally implantable in human bodies. In terms of the size, the OD of the tube cannot be bigger than 690 $\mu \mathrm{m}$ and ID should be larger than $500 \mu \mathrm{m}$. The length of the tube is trimmed to $6 \mathrm{~mm}$ long to fit in the eye. Both ends of the tube are slanted with 30 degree for easy implantation.

\section{Design of parylene anchor}

The anchor is made of parylene $\mathrm{C}$, with rollable swings that are squeezed into hypodermic needle during implantation and rolled out afterward. A trench with $300 \mu \mathrm{m}$ in radius is designed to accommodate the parylene micro tube. The overall length is designed as $5 \mathrm{~mm}$, which is shorter than the tube so that the slanted end of the tube can be exposed during implantation.

\section{DEVICE FABRICATION}

\section{A. Fabrication of NC check valves}

The main fabrication processes are shown in Figure 5. The fabrication started with growing thermal oxide on silicon surface. DRIE was then performed to define the location of orifices and the boundary of valves on the back side. $\mathrm{XeF}_{2}$ was used to roughen the front side surface to help parylene anchoring. 3-step lithography was done to create 3 different photoresist heights. Parylene was then coated, patterned, and the orifices were opened by DRIE on the back side again. Photoresist was stripped by soaking in the acetone and the whole valve can be released manually when in need.

\section{B. Fabrication of parylene micro tubes and anchors}

There are two ways to manufacture parylene micro tubes. One choice is to coat parylene directly onto both inside and outside of commercialized glass tubes. The other way is to coat only on the outer surface. Although, for the former approach, it may be biocompatible by coating a layer of parylene on the glass, it still remains very brittle and is highly possible to break inside the eye or during the surgery. Therefore we chose the latter approach that coats parylene only on the outer surface of glass tubes and released the glass inside the parylene micro tubes by soaking tubes long in BHF. The thickness of the tube is another concern in terms of implantation. It can neither be too thin nor too thick. Thinner parylene tends to be crashed in the eye due to the muscle pressure and thicker parylene becomes difficult to fit in a small gauge needle.

The fabrication of parylene anchors start with $\mathrm{XeF}_{2}$ silicon isotropic etching using oxide as the etching mask. After oxide mask removed, the front side silicon surface was coated with a layer of parylene and then aluminum. A layer of dry film photoresist was then laminated on top of the trenches and patterned, which becomes the mask for aluminum etching. Parylene was later patterned using etched aluminum layer and the whole parylene anchor can be released by soaking in DI water with the help of ultrasonic. 


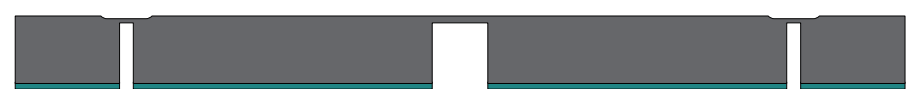

1. Two side oxide growing

2. Back side lithography, $\left(1^{\text {st }}\right.$ mask $)$

3. Back side oxide patterning and DRIE

4. Front side $\mathrm{XeF}_{2}$ surface roughening, ( $2^{\text {nd }}$ mask)

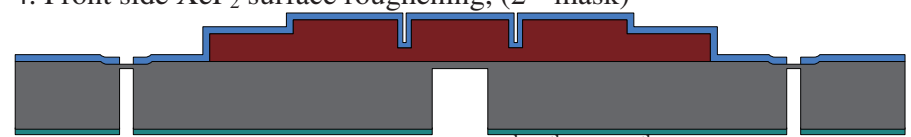

1. Front side three-steps lithography, $\left(3^{\text {rd }}, 4^{\text {th }}\right.$ and $5^{\text {th }}$ masks $)$

2 . Front side parylene coating and patterning, $\left(6^{\text {th }}\right.$ Mask $)$

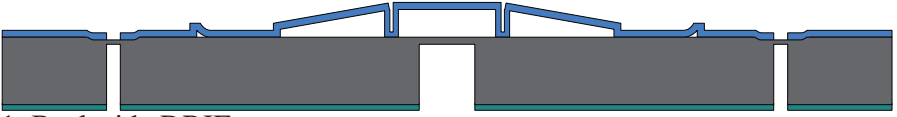

1. Back side DRIE

2. Photoresist stripping and stiction happens afterwards

Silicon $\square$ Oxide $\square$ Parylene $\square$ Photoresist

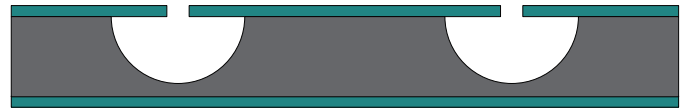

1. 2 sides oxidation and patterning, ( $1^{\text {st }}$ mask)

2. XeF2 isotropic etching

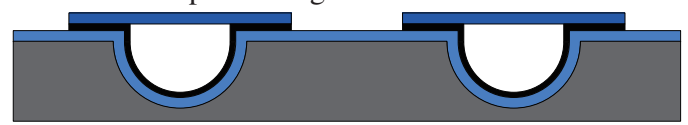

1. Oxide removing

2. Parylene coating

3. Aluminum deposition

4. Dry film photoresist lamination and patterning ( $2^{\text {nd }}$ mask)

5. Aluminum etching
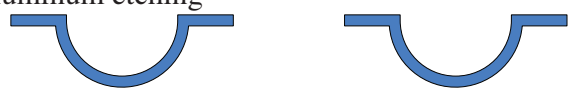

1. Parylene RIE etching

2. Aluminum stripping

3. Parylene releasing

Figure 5. Fabrication process of (left) NC check valves and (right) parylene anchors

(a)
Chip before

(b)

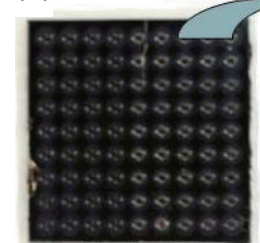

Photoresist stripping Single valve releasing

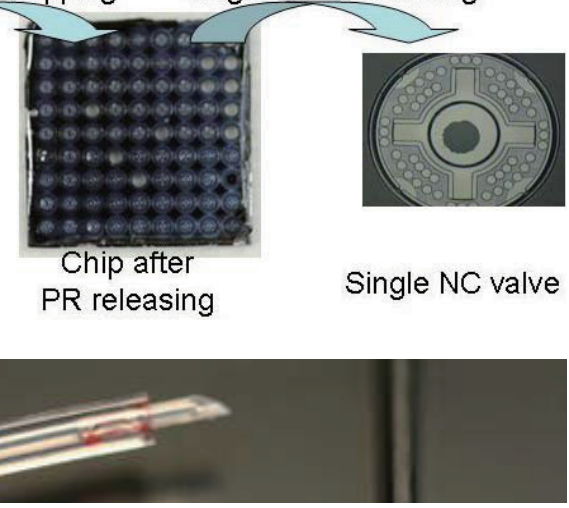

(c)

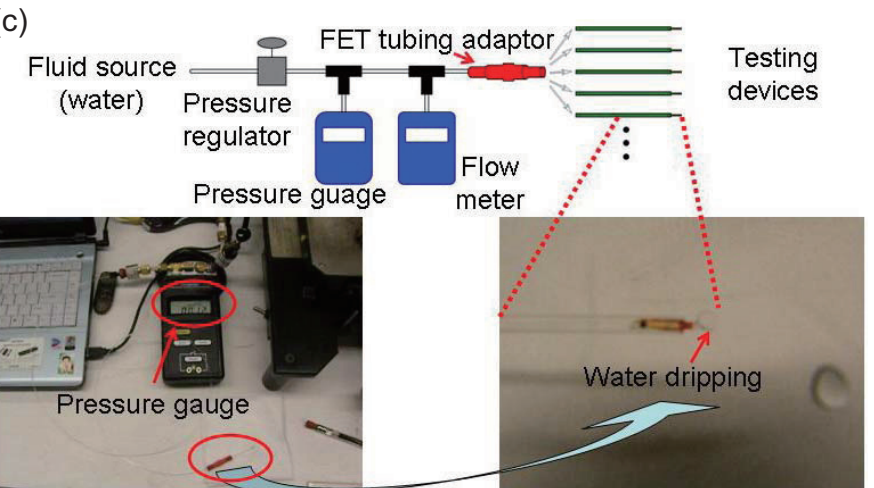

(d)

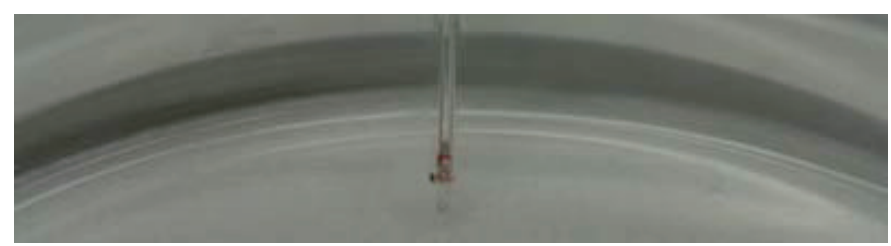

Figure 6. Pre-implanted preparation procedures: (a) releasing NC check valves from a chip; (b) packaging valve within a tube and sealing the valvetube with photoresist in another capillary tube; (c) in vitro testing; (d) releasing the valve-tube by acetone and IPA and attached onto parylene fixation anchors.

\section{DEVICE PACKAGING AND BENCH-TOP TESTING}

The chip with valves was first dipped into acetone, IPA and DI water to strip off protective photoresist. Each valve is released from the chip by breaking silicon connection part as shown in Figure 6. The NC check valve was then inserted with parylene side facing the interior of the parylene micro tube in order to prevent damaging the parylene membrane during implantation. The slit between the valve and the parylene micro tube was completely sealed by epoxy. After epoxy dried, the valve-tube assembly was inserted into DuPont Teflon FET capillary tube, and photoresist was used to seal the gap between FET tube and valve-tube structure. The FET tube was cut into 2 inch segments in advance and the integrated valve-tube assembly was attached to one end of the capillary tube. The whole setup was baked to dry in a convectional oven at $100{ }^{\circ} \mathrm{C}$. After photoresist dried, each assembly was connected to the testing setup shown in Figure 6. The bench-top testing setup used compressed nitrogen to applied pressure to water. The setup also contains one pressure regulator and one pressure gauge. Water is used as working fluid to mimic the eye fluid. The flow rate is calculated by measuring the marching speed of the moving water front. The characteristic curve of every assembly was generated and also filmed. In order to get a qualified working device, the result of the cracking pressure is required to fall in between $10-20 \mathrm{mmHg}$. It's also required that water drips should only be observed flowing out through the opening of valve-tubes, not the slit between valve-tubes and the FET tubes. In vitro testing results are shown in TABLE I. Tested and qualified valve-tube devices were then released by acetone and IPA and attached to a parylene fixation anchor.

TABLE I. IN VITRO AND EX VIVO CRACKING PRESSURE RESULTS:

\begin{tabular}{|c|c|c|}
\hline Measured Cracking Pressure (mmHg) & In vitro & Ex vivo \\
\hline GDD 1 & 17 & $15-25^{*}$ \\
\hline GDD 2 & 12 & 24 \\
\hline
\end{tabular}

*Number is obtained by estimation from video timeline. 


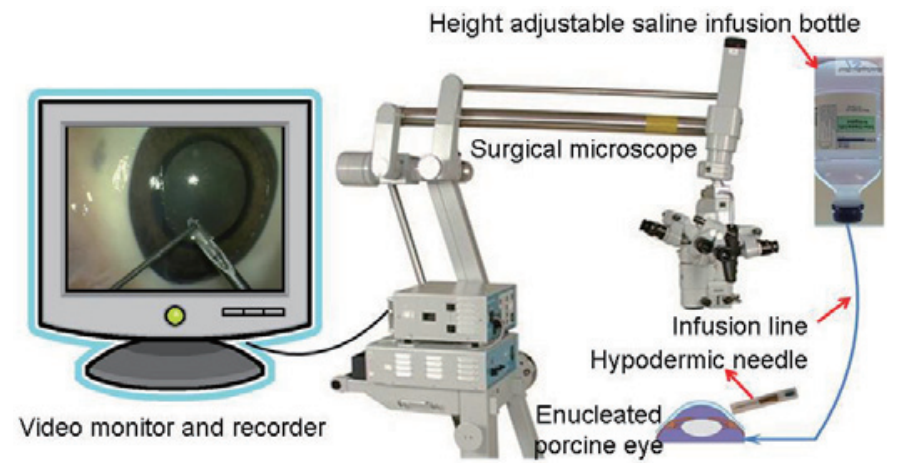

Figure 7. Testing setup of ex vivo implantation.

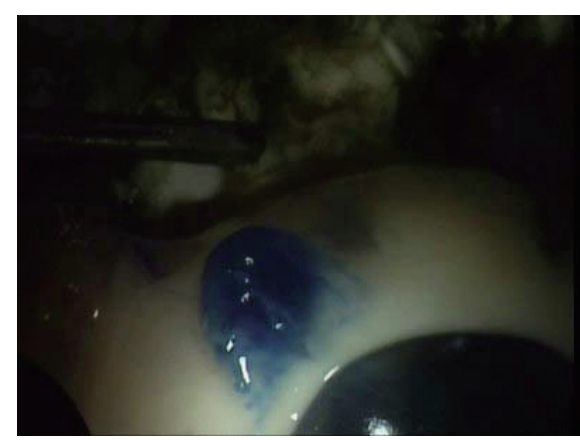

Figure 8. Ex vivo implantation result of GDD 1.

(a)

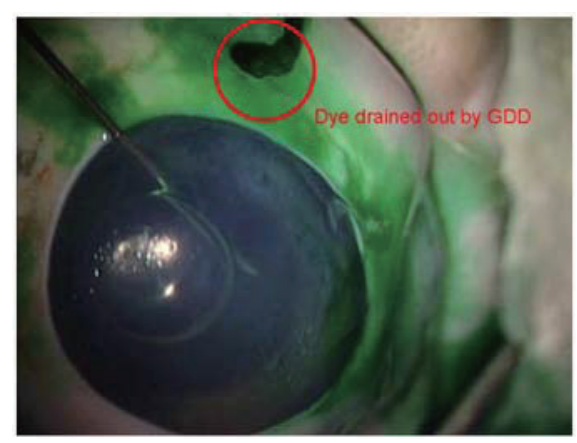

(b)

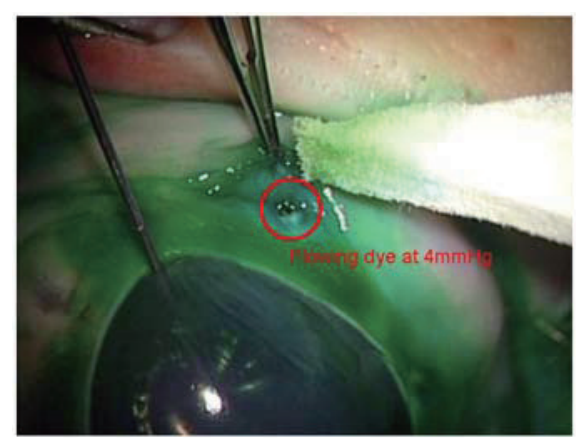

Figure 9. Ex vivo implantation results of GDD 2: (a) Dye started to drain out after fluids injected and stopped at $24 \mathrm{mmHg}$; (b) Residual dye remained slightly flowing at $4 \mathrm{mmHg}$.

\section{EX VIVO IMPLANTATION AND DISCUSSION}

A gauge \#19 hypodermic needle and a blunt end plunger are used as the GDD carrier and deliverer, respectively. Two ex vivo implantations are performed to understand the behavior of the integrated GDD implanted in enucleated porcine eyes and the function of the parylene fixation anchor. The ex vivo implantation setup is shown in Figure 7., with saline as the working fluid. It can be shown that parylene fixation anchor helps the GDD stay firmly without moving successfully after needle retracted. The ex vivo testing results are also summarized in TABLE I. The infusion line was connected to the vitreous chamber so that different pressures can be provided by raising the infusion bottle. After implantation, a blue dye was injected into the anterior chamber, then the infusion bottle was raised up to increase the pressure. Soon after the infusion line was turned on, the dye was seen to come out of the GDD, as shown in Figure 8. After a few seconds, when the IOP reaches $52 \mathrm{mmHg}$, the infusion line is turned off. The video shows that GDD successfully opened to drain out the dye well before the IOP reached its final $52 \mathrm{mmHg}$. From the timeline of the video, the turn-on pressure is around 15-25 $\mathrm{mmHg}$.

To understand more about the behavior of the external end of the GDD, another implantation was performed with one end of the GDD exposed to air. Unlike the previous setup, the infusion line and the dye were both inserted and injected directly into anterior chamber to mimic the accumulation of aqueous humor during real glaucoma. Video recordings show that once saline and dye were both injected into the anterior chamber, the GDD started to drain out the liquid, as shown in Figure 9. We adjusted the saline flow rate and observed that the GDD stopped to drain out fluids at $24 \mathrm{mmHg}$. The offset of the cracking pressure is possibly due to differences between in vitro and ex vivo testing environments. A hysteresis study was also executed at the end of the test to quantify the lowest pressure required to keep the GDD open. Using a cotton swab, capillary forces were introduced to continually pull eye fluids out of the GDD. It was observed that the dye flow continued until the pressure reached $4 \mathrm{mmHg}$ IOP for a few seconds. This result indicates that the response time of the valve's restoring force could be as long as several seconds. After experimentation, the implanted GDD was retracted and investigated. More detailed hysteresis behavior study is also underway.

\section{ACKNOWLEDGEMENT}

The authors would like to thank Dr. Trevor Ropers technical assistance in terms of device fabrication.

\section{REFERENCES}

[1] Glaucoma Research Foundation, http://www.glaucoma.org/

[2] J. C.-H. Lin, P.-J. Chen, S. Saati, R. Varma, M. Humayun, Y.-C. Tai, "Implantable Microvalve-Packaged Glaucoma Drainage Tube", in Tech. Digest 13th Solid State Sens., Actuators, and Microsyst. Workshop, Hilton Head, SC, Jun. 1-5, 2008, pp. 146-149.

[3] J. C.-H. Lin, P.-J. Chen, B. Yu, M. Humayun, Y-C. Tai, "Minimally Invasive Parylene Dual-Vavled Flow Drainage Shunt for Glaucoma Implant", in Tech. Digest 22nd IEEE International Conference on MicroElectroMechanical Systems (MEMS 2009), Sorrento, Italy, Jan. 25-29, pp.196-199.

[4] P.-J. Chen, D. C. Rodger, S. Saati, J. C. Altamirano, C.-H. Lin, R. Agrawal, R. Varma, M. S. Humayun, and Y.-C. Tai, "Implementation of Microfabricated Sutureless Flexible Parylene Tissue Anchors on Minimally Invasive Biomedical Implants", in Proc. MicroTAS 2007, Paris, France, pp. 518-520. 